Reference was made to the possible connexion between the molecular structure and the exhibition of anisotropic properties, and to the various theories that have been advanced to explain the peculiar properties of these somewhat unfortunately named liquids.

Thursday's activities were inaugurated by a lecture by the Master of Trinity on "The Electron in Chemistry." Sir J. J. Thomson apologised, as a physicist, for encroaching on the domain of the chemist, but added that the difference between chemistry and physics was due to want of knowledge, and that the problem of chemical combination was one of the most outstanding problems in physics. Dalton's Atomic Theory, as such, took no account of the intrinsic structure of discrete particles, and the modern conception of the internal arrangements of the atom dated from the discovery of the electron in I897. The necessity of postulating a central positive nucleus and the possible arrangements of electrons around this was then discussed, and with the aid of diagrams and data thrown on the screen Sir Joseph reviewed existing knowledge of atomic structure, adequately deduced the existence of two forms of nitrogen, and showed that electrostatic considerations limited the number of electrons in a stable ring to eight. The latter part of his paper was devoted to the fascinating but somewhat intricate problem of chemical combination and the idea of " activated " molecules.

In addition to these lectures members of the Association divided to hear the very interesting and amusing lecture on "The Acoustics of Public Buildings," by Mr. A. Wood, and a lecture, equally attractive in its illustration, on "Coral Reefs in the Pacific," by Mr. F. A. Potts.

The scientific interests of the members were further selectively absorbed on Thursday by a lecture in the Anatomical Department by Dr. H. Hartridge on "Physiological Limits to the Accuracy of Visual Measurements "- -a lecture of great interest to physicists among others-proceeding simultaneously with a lecture by Mr. E. K. Rideal on "Molecular Orientation on Plane Surfaces ": in this, interesting deductions were made from the assumption that surface energy effects are restricted to a film of unimolecular thickness.

The visitors to the Cavendish Laboratory enjoyed Dr. Searle's demonstration of novel methods of determining physical quantities as well as the exhibit of apparatus used by Maxwell, Raleigh, Kelvin, Stokes, and other pioneer physicists. Prof. Marr prefaced his conducted tour of the Sedgwick Museum by a short lecture on some geological considerations suitable for school treatment, while demonstrations of great interest to those engaged in the teaching of science were set up in the laboratories devoted to chemistry, physical chemistry, metallurgy, botany, physiology, experimental psychology, zoology, mineralogy, and in the new Department of Engineering.

A conversazione in the Large Examination Hall on Thursday evening officially terminated the meeting (although the laboratories were opening on the Friday to provide further opportunities for those desiring to visit them): on this occasion Mr. R. E. Priestley lectured on "Antarctic Exploration with Shackleton and Scott." 'Mr. Priestley's amusing and thrilling lecture, accompanied by lantern illustration that won frequent applause, provided an appropriate conclusion to a richly stimulating meeting.

It remains to be mentioned that well-known firms held an exhibition of books and apparatus in the Arts School.

\section{Association of Women Science Teachers.}

At the annual meeting of the Association of Women Science Teachers held at University College, London, on January 6 , a report was received from the subcommittee appointed to investigate the possibility of getting into touch with Colonial and foreign teachers of science. An appeal was made for members to correspond with teachers in other countries, and especially to send scientific journals to them. It is hoped that this movement may be further developed and become a useful part of international co-operation.

In her presidential address Miss M. B. Thomas reviewed the criticisms which have recently been made against methods of teaching science in schools. She pointed out that it was impracticable, under existing conditions, to combine preparation for university entrance examinations with the wide and more generalised scientific instruction which was so generally felt to be desirable, and pleacled for greater co-ordination between the subjects taught in schools. It was obvious that Science, and Languages, English, etc., could be mutually helpful, and that a closer co-operation between the mistresses teaching these subjects would result in advantage to all the subjects.

In the afternoon a large and appreciative audience heard a lecture by Dr. Dorothy Wrinch on " Relativity and Scientific Method." The lecturer gave an exposition of this difficult subject which was so clear that even her non-mathematical hearers could follow the argument. She pointed out that the old dynamics had rested entirely upon the idea of measurement relative to a rigid and stationary standard, and that if the standard moved with a uniform velocity the position of affairs was altered. Examples were quoted in which the new equation for the composition of velocities has solved long-standing problems. Dr. Wrinch then proceeded to apply the principle to various kinds of scientific problems, which must not be approached on the assumption that the old laws would hold good but with the possibility in view that some law of the same nature as that of relativity might be the governing principle. To sum up, it must be remembered that if such apparent fundamentals as time and distance have been shown to depend on velocity, then velocity is a relevant variable in all scientific method.

\title{
Hail and Sleet in Meteorological Terminology.
}

$A \mathrm{~T}$ intervals there appears in the meteorological literature of various countries a discussion concerning the proper designation of the smaller and softer forms of hail which are common in all European countries during the winter or spring months. A recent contribution to the subject by $\mathrm{R}$. Giacomelli, appearing in the issue for May and June of La Meteorologia pratica, the organ of the observatory of Montecassino, near Naples, is illuminat- ing from certain points of view, without really settling the question. It is pointed out that the French and German terms, grésil and gräupel respectively, have the root idea of little pellets or grains, and that the real Italian equivalent, gragnola, is meteorologically a better descriptive term since it means " little hail." In full keeping, moreover, with the almost amusing richness of the Italian language in diminutive terms, one may use in place of gragnola 
the words granzola, granuschia, gragnolischia, all of which are derived from grandinola (grandine, hail), and each of which is locally favoured in various parts of central Italy, where such forms of frozen precipitation are fairly frequent in the spring period, March and April.

In English we have no distinctive word, nor, as it is hoped to show in this note, do we really need one. The familiar word "sleet" appears to follow the German Schlacken in denoting a mixture of rain and snow in the British Isles; but in the United States "slect" is officially reserved for true frozen rain, that is to say, drops which congeal into clear ice spherules by passing through a cold surface stratum of air. This kind of hail, as one would categorise it in England, is a common winter phenomenon in the eastern States, because there the contrasts of temperature between the equatorial and polar currents in cyclones, though not more frequent than in England, are more violent, so that a warm rain more often than here alights on a frozen soil. But, on the other hand, various forms of wintry hail falling in showers in moderately cold polar currents during the winter and spring are distinctly common in England, and these show almost every gradation from the little soft white opaque pellets, which are really hardened snowflakes and might be called " snow-hail," to something very like the real hail more typically associated with summer thunderstorms. It is clear that ambiguity would arise if "sleet" were used for any of these forms in this country. In fact, the British official practice of comprising all forms of frozen precipitation other than snow under the term "hail" is philosophically sound, and no regret need really be felt that we have no word to correspond to grésil, gräupel, or gragnola. It would appear that the only real solution of this terminological difficulty is to recognise but three fundamental species of precipitation: "rain," the liquid form; "snow," the frozen form in flakes or dust; and " hail," the frozen form in stones or pellets.

Actually, the different varieties of hail scarcely differ more from one another than do the different varieties of snow, or even of rain, and no difficulty need be felt on that score. Doubtful forms, such as the "snow-hail" referred to above, had best be entered in a register to both species; and in the case of the mixture of rain and snow, which in this country we call "sleet," this is habitually done. The double-entry plan has the advantage of tending to eliminate the effect of personal bias on the part of an observer, a factor which probably affects quite seriously the comparability of snow-frequency statistics in different localities.

\section{The International Astronomical Union.}

V OLUME I. of the Transactions of the International Astronomical Union, giving an account of the first general assembly held at Rome, May last, is edited by Prof. A. Fowler (London: Imperial College Bookstall, Prince Consort Road, S.W.7 ; price $x_{5}$ s.). It is an indispensable book of reference for astronomical workers, which contains the agenda of the thirty-two commissions, including important proposals for the co-ordination of methods of observing and mapping out of the fields of work to avoid useless duplication. The spectroscopic data are particularly full: the Draper spectral notation has been slightly modified and considerably extended in the light of increased knowledge. The letter $Q$ is assigned to novæ, and the well-known stages in the development of the nova spectrum are indicated by suffixes. There is also a list of wave-lengths of iron, neon, and other lines suggested as standards.

Some of the decisions may be given briefly. The Latin names of the constellations are to be used, and a set of 3-letter abbreviations of these names was agreed to. The kilometre is to be used for lineof-sight velocities and for dimensions of bodies, the astronomical unit for planetary distances, the parsec for stellar distances. Absolute magnitude is defined as the magnitude at a distance of ro parsecs. Certain letters were formerly used with several different meanings; they are now distinguished thus: $[\mathrm{A}]=\mathrm{a}$ line in the spectrum, $\underline{\mathrm{A}}$ or $A$ (ital.) $=$ Argon, $\mathrm{A}=\mathrm{a}$ stellar spectral type.
The Conn. des Temps list of Fundamenual stars and the Carte $d u$ Ciel list of intermediary stars were adopted as standards, and $1925^{\circ} \mathrm{O}$ is to be used as the standard equinox up to I940. M. Andoyer undertook to reduce the latest positions of the fundamental stars to this equinox.

It was recommended that the short-period variations in solar radiation, announced by Abbot, should be studied as widely as possible, and their correlation with weather changes investigated.

Photometric work on minor planets was recommended. In stellar-parallax work it was recommended that plates of each field should be repeated after Io years, to obtain the proper motions of the comparison stars.

A central bureau for double-star work was recommended, and various decisions for securing uniformity of method were passed. A variable star bureau or centre in each country is desirable (one has been established at Lyons). The Cracow Observatory undertakes the preparation of ephemerides of Algol stars.

The commission on calendar reform recommended (x) a perpetual calendar, with a 52 -week year and one or two days outside week and month, (2) the lengths of the months in each quarter should be 30,30 , 3 I days, and (3) that the year should begin at the winter solstice.

The volume is thus a noteworthy record of important decisions, embracing nearly every branch of astronomy.

\section{The Haber Process.}

THE lecture delivered by Prof. F. Haber on the award of the Nobel Prize at Stockholm on June I, I920, is printed in Die Naturwissenschaften for December 8. Prof. Haber dealt first with the work done on the synthesis of ammonia before his first research in I905. Practically nothing of importance had come to light, and the very small yields at ordinary pressures did not hold out much promise of technical application.

The early experiments of Haber, like most of those which have served as the foundations of great industrial undertakings, were made with a purely scientific object, and with no technical applications in view. The results obtained, however, soon made it clear that the basis of an important technical process could be found in ammonia synthesis, and further work was undertaken with this end in sight.

In 1908 the Badische Gesellschaft placed at Haber's disposal all the means requisite for the further progress of the research on the synthesis of nitric oxide 